\title{
CHARACTERIZATION OF REACTIVELY R.F.-SPUTTERED TANTALUM OXIDE FILMS
}

\author{
YUAN-KUANG TU* AND CHIA-CHIEN LIN \\ Telecommunication Laboratories-Ministry of Communications, P.O. Box 71, Chung-Li 32099 (Taiwan) \\ WAY-SEEN WANG \\ Department of Electrical Engineering, National Taiwan University, Taipei 10764 (Taiwan) \\ SHENG-LIANG HUANG \\ Department of Physics, National Tsing Hua University, Hsin-Chu 30043 (Taiwan) \\ (Received January 7, 1988; accepted February 8, 1988)
}

The characteristics of reactively r.f.-sputtered tantalum oxide films formed under a variety of sputtering conditions have been studied. Sputtering gas composition, gas pressure, r.f. power and substrate temperature have been varied to study their effects on the refractive index, deposition rate, film structure and atomic composition of the deposited tantalum oxide films. For a certain total pressure and gas composition of the reactive atmosphere, the deposition rate and refractive index will reach a peak value. An increase in the r.f. sputtering power will increase the deposition rate, but the refractive index of the deposited films will be decreased. An increase in the substrate temperature will lead to films of higher refractive indices. Correlations between the atomic composition and the refractive index have been studied and it is found that, the larger the atomic ratio of oxygen to tantalum, the higher is the refractive index.

\section{INTRODUCTION}

Tantalum oxide films have been extensively used as the dielectrics of thin film capacitors, insulators, antireflection coating materials ${ }^{1,2}$ and optical dielectric waveguides $^{3-5}$. The tantalum oxide films are quite durable chemically and mechanically with a very low optical attenuation loss and a relatively high refractive index, so they have been considered to be one of the best materials for the fabrication of optical waveguides and lenses and for the improvement of the acousto-optic interaction effects on lithium niobate diffused waveguides 5 . Tantalum oxide films can be deposited by several techniques, such as anodization and thermal oxidation from the deposited tantalum films, reactive sputtering from a tantalum (or tantalum oxide) target and chemical vapour deposition ${ }^{1,2}$. It is known that tantalum oxide films with the lowest optical loss can be obtained by the reactive sputtering technique ${ }^{4,5}$. In this study, the reactive sputtering technique was also employed to deposit the tantalum oxide films as the optical waveguide material. However, from the atomic ratio of oxygen to tantalum obtained from the Rutherford backscattering spectrometry (RBS) analyses, it is found that this ratio is less than 2.5 , which is

\footnotetext{
*Also with the Department of Electrical Engineering, National Taiwan University, Taipei 10764, Taiwan.
} 
different from the previously reported value ${ }^{4,5}$. For a deposited tantalum oxide film that can be used as a useful waveguide material, it is then important and also is the purpose of this study to investigate the relationships of the deposition parameters (gas composition, total pressure, r.f. power, substrate temperature etc.) with the film's refractive index, deposition rate, film structure and atomic composition.

\section{SAMPLE PREPARATION AND MEASUREMENT}

In this study, tantalum oxide films were grown using the reactive sputtering technique. An R\&D type of r.f. diode sputtering system with a 4 in diameter tantalum target was used in the investigation. The sputtering process was carried out in a reactive oxygen-argon atmosphere. Both silicon wafers and Corning 7059 glass sheets were chosen as the substrates for growing the desired tantalum oxide films. Samples on silicon wafers were prepared for the measurement of the refractive index and the thickness of the deposited films and also for the characterization of the microstructure and atomic composition. Samples on glass substrates were prepared to study their optical properties. The sputtering deposition parameters, such as sputtering gas composition, gas pressure, r.f. power and substrate temperature, were carefully adjusted and varied to investigate the effects on the refractive index, film deposition rate, microstructure, atomic composition and optical properties of the deposited tantalum oxide films. For each sputtering process, well-cleaned and dried substrates were loaded onto the substrate holder, and the system was evacuated to a vacuum below $5 \times 10^{-6}$ Torr. Then the flow rates of the argon and the oxygen gases were adjusted by separate flowmeters and introduced into the chamber. The sputtering gas pressure was controlled by a throttle valve between the vacuum chamber and the diffusion pump.

To investigate the effects of oxygen contents (the ratio of the oxygen partial pressure to the total gas presssure was defined as the oxygen content), the argon partial pressure was maintained at $7 \times 10^{-3}$ Torr, while the oxygen partial pressure was varied so that the oxygen content ranged from $2.78 \%$ to $50 \%$. The effects of the total sputtering gas pressure were studied by adjusting the throttle valve so as to change the sputtering pressure (varied from $4 \times 10^{-3}$ to $25 \times 10^{-3}$ Torr) but with a constant oxygen content $(16.7 \%$ in this case). In the experiments above, the r.f. power was kept at $200 \mathrm{~W}$ and the substrate temperature was set at $25^{\circ} \mathrm{C}$.

To understand the individual effects of the substrate temperature and the r.f. power, the substrate temperature was allowed to vary from 25 to $300^{\circ} \mathrm{C}$ by using a substrate heater monitored by an attached thermocouple. During the experiment, the total gas pressure was $8.4 \times 10^{-3}$ Torr, the oxygen content was $16.7 \%$, and the r.f. power was $200 \mathrm{~W}$. The r.f. sputtering power, ranging from 100 to $250 \mathrm{~W}$, was controlled directly from the output of the r.f. generator. Samples for this study were prepared with different oxygen contents $(5.40 \%$ and $31.4 \%)$ while keeping the argon pressure constant $\left(7 \times 10^{-3}\right.$ Torr $)$ and the substrate temperature at $25^{\circ} \mathrm{C}$.

Both the refractive index and the thickness of the deposited tantalum oxide film were measured by an ellipsometer with an $\mathrm{He}-\mathrm{Ne}$ laser $(633 \mathrm{~nm})$ source. The microstructure of the deposited films was investigated by X-ray diffractometric (XRD) measurements, and the atomic composition of the films was studied by RBS 
analyses. Finally, the prism coupling method was used to study the optical properties of the deposited tantalum oxide films.

\section{RESULTS AND DISCUSSION}

From the XRD measurements, as shown in Fig. 1, it is found that all the samples prepared in this study are amorphous. The RBS analyses show that the atomic compositions of the deposited tantalum oxide films vary with different deposition parameters, and argon atoms are found in all the deposited samples. $A$ typical RBS analysis profile is shown in Fig. 2. The variations in the atomic

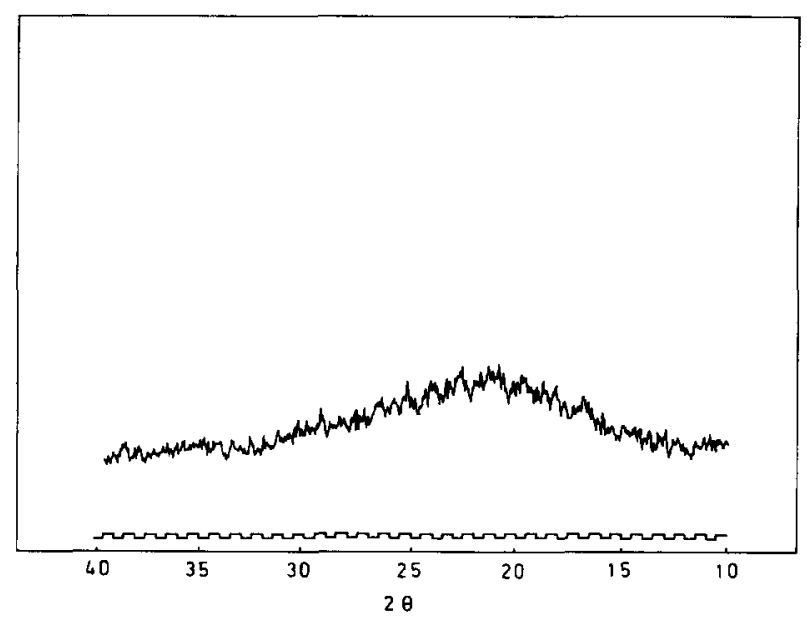

Fig. 1. X-ray diffraction pattern of the deposited tantalum oxide film for a total pressure of $8.4 \times 10^{-3} \mathrm{Torr}$, an oxygen content of $16.7 \%$, an r.f. power of $200 \mathrm{~W}$ and a substrate temperature of $300^{\circ} \mathrm{C}$.

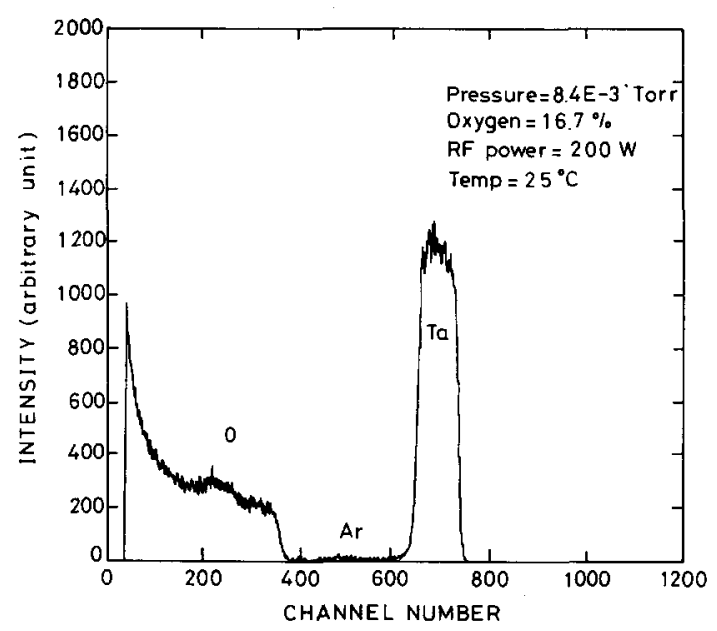

Fig. 2. A typical RBS analysis profile of the deposited tantalum oxide film. 
compositions of the deposited tantalum oxide films with various deposition conditions are shown in Tables I-III. From these tables it can be seen that the tantalum oxide films prepared in this study are not in the stoichiometric $\mathrm{Ta}_{2} \mathrm{O}_{5}$ structure.

TABLE I

ATOMIC RATIOS OF OXYGEN TO TANTALUM IN THE TANTALUM OXIDE FILMS FOR VARIOUS OXYGEN CONTENTS

\begin{tabular}{llccc}
\hline Oxygen content $(\%)$ & 2.78 & 16.7 & 31.4 & 50.0 \\
Atomic ratio $[\mathrm{O}] /[\mathrm{Ta}]$ & 2.136 & 2.273 & 2.248 & 2.065
\end{tabular}

Argon pressure, $7 \times 10^{-3}$ Torr; r.f. power, $200 \mathrm{~W}$; substrate temperature, $25^{\circ} \mathrm{C}$.

TABLE II

ATOMIC RATIOS OF OXYGEN TO TANTALUM IN THE TANTALUM OXIDE FILMS FOR VARIOUS TOTAL PRESSURES

\begin{tabular}{lllc}
\hline Total pressure (mTorr) & 4.0 & 8.4 & 25.0 \\
Atomic ratio $[\mathrm{O}] /[\mathrm{Ta}]$ & 2.123 & 2.273 & 2.081 \\
\hline
\end{tabular}

Oxygen content, $16.7 \%$, r.f. power, $200 \mathrm{~W}$; substrate temperature, $25^{\circ} \mathrm{C}$.

TABLE III

ATOMIC RATIOS OF OXYGEN TO TANTALUM IN THE TANTALUM OXIDE FILMS FOR VARIOUS SUBSTRATE TEMPERATURES

\begin{tabular}{lllll}
\hline Substrate temperature $\left({ }^{\circ} \mathrm{C}\right)$ & 25 & 100 & 180 & 200 \\
Atomic ratio $[\mathrm{OO}] /[\mathrm{Ta}]$ & 2.273 & 2.356 & 2.423 & 2.481 \\
\hline
\end{tabular}

Total pressure, $8.4 \times 10^{-3}$ Torr; oxygen content, $16.7 \%$ r.f. power, $200 \mathrm{~W}$.

By varying the oxygen content while keeping the argon partial pressure constant $\left(7 \times 10^{-3}\right.$ Torr), as shown in Fig. 3 , both the refractive index and the corresponding deposition rate of the deposited films show a peak value at an oxygen content of $16.7 \%$ (the total pressure is $8.4 \times 10^{-3}$ Torr). It can be seen from this figure, that, with the argon partial pressure at $7 \times 10^{-3}$ Torr and the oxygen content below $16.7 \%$, the sputtering yield and hence the deposition rate will increase with the

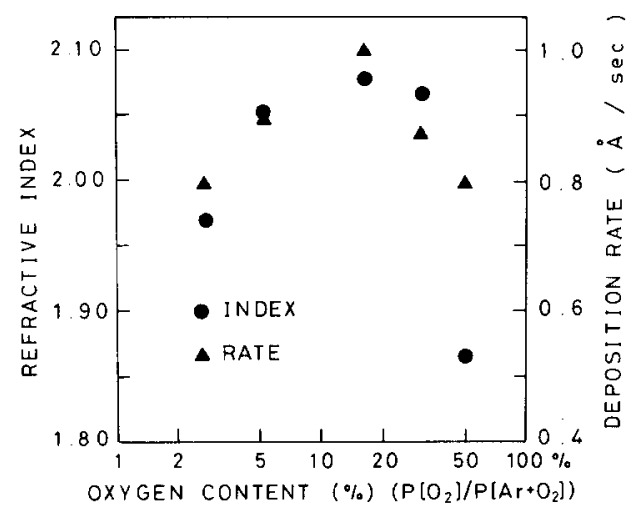

Fig. 3. Refractive index and deposition rate $v$ s. oxygen content (argon partial pressure, $7 \times 10^{-3}$ Torr; r.f. power, $200 \mathrm{~W}$; substrate temperature, $25^{\circ} \mathrm{C}$ ). 
oxygen content and so will the refractive index. These phenomena arise because an increase in the oxygen content (but still below 16.7\%) will lead the system to operate closer to its optimal condition $\left(8.4 \times 10^{-3}\right.$ Torr $)$ and so the sputtering yield and the deposition rate will increase. The increase in the oxygen content will enhance the reaction probability between the oxygen and the sputtered tantalum atoms, and it follows that the composition of the deposited tantalum oxide films becomes oxygen rich, which has been verified from the RBS results (Tables I-III). However, as can be seen from Fig. 3, when the oxygen content is greater than $16.7 \%$, both the deposition rate and the refractive index become smaller. This is due to the excess oxygen, which results in surface oxidation of the target and then the degradation of the sputtering yield.

Figure 4 shows the variation in the refractive index and the deposition rate with the total gas pressure while the oxygen content was kept at $16.7 \%$. As can be seen from this figure, the deposition rate has a maximum value at a total gas pressure of $8.4 \times 10^{-3}$ Torr and decreases at lower or higher total pressures. Also, a drastic drop in the refractive index is observed when the total gas pressure is above $15 \times 10^{-3}$ Torr. This is because of the decrease in the particle mean free path and then in the sputtering yield at the higher total pressure.

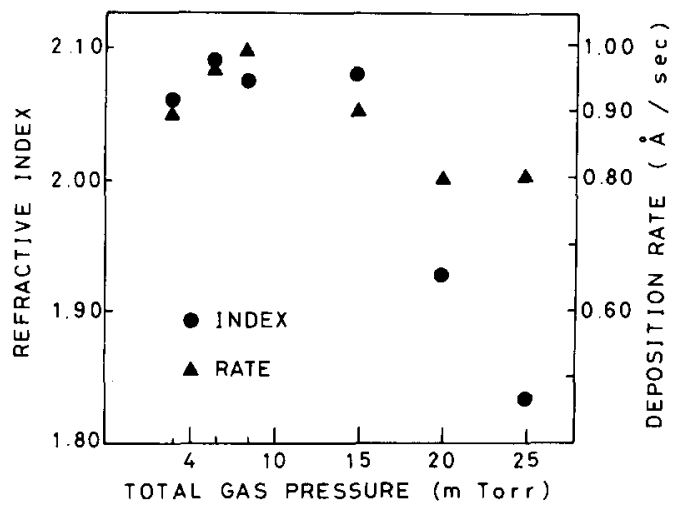

Fig. 4. Refractive index and deposition rate $v$ s. total pressure (oxygen content, $16.7 \%$; r.f. power, $200 \mathrm{~W}$; substrate temperature, $25^{\circ} \mathrm{C}$ ).

When the r.f. sputtering power is increased while keeping the argon pressure at $7 \times 10^{-3}$ Torr and for oxygen contents of $5.40 \%$ and $31.4 \%$, as shown in Fig. 5 , the deposition rate increases while the refractive index decreases. This is because the sputtering yield will increase with the r.f. sputtering power and so will the deposition rate; therefore, the reactions between the sputtered tantalum atoms and oxygen atoms, under a fixed oxygen content, are changed so that the composition of the deposited tantalum oxide film becomes tantalum rich, which results in a decrease in the refractive index.

Figure 6 shows the variations in both the refractive index and the deposition rate $v s$. the substrate temperature, with a total gas pressure of $8.4 \times 10^{-3}$ Torr and an oxygen content of $16.7 \%$. It is found that the refractive index increases with the substrate temperature but the deposition rate is almost constant. When the 


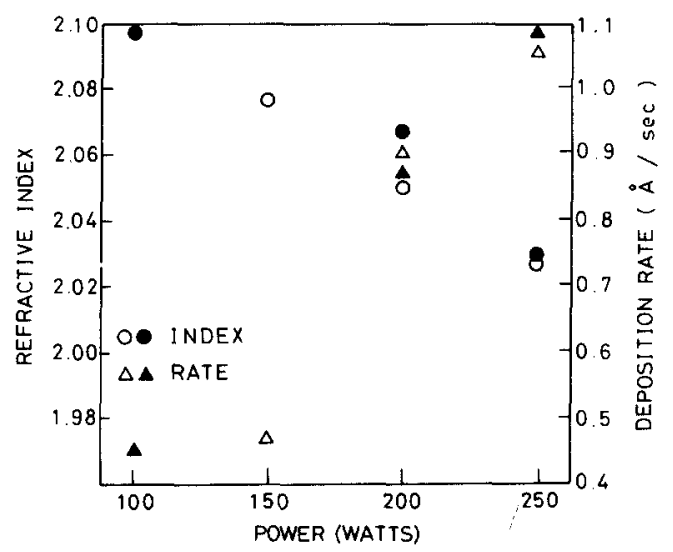

Fig. 5. Refractive index and deposition rate vs. r.f. sputtering power (argon partial pressure, $7 \times 10^{-3}$ Torr; oxygen contents, $5.40 \%(O, \Delta)$ and $31.4 \%(\bullet, \Delta)$; substrate temperature, $\left.25^{\circ} \mathrm{C}\right)$.

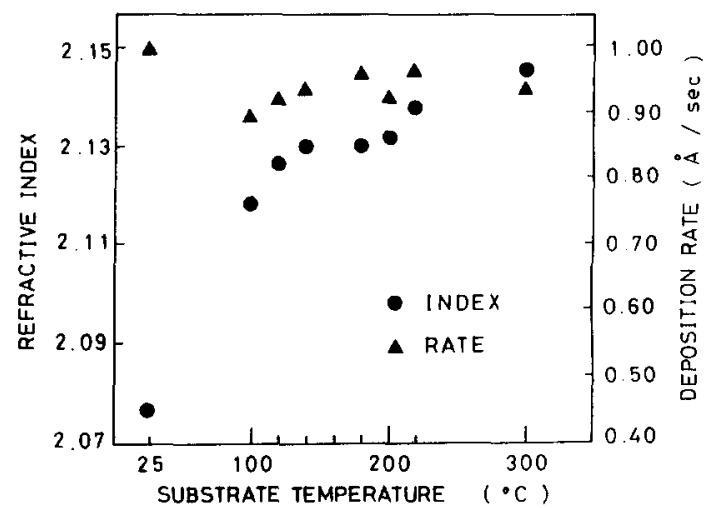

Fig. 6. Refractive index and deposition rate vs. substrate temperature (total gas pressure, $8.4 \times 10^{-3} \mathrm{Torr}$; oxygen content, $16.7 \%$; r.f. power, $200 \mathrm{~W}$ ).

substrate temperature is increased, the structure of the tantalum oxide film becomes more stoichiometric, as can be seen from Table III, the atomic ratio of oxygen to tantalum increases from 2.273 at $25^{\circ} \mathrm{C}$ to 2.481 at $200^{\circ} \mathrm{C}$, and the corresponding refractive index becomes higher.

Comparing Tables I-III with Figs. 3, 4 and 6, we find that the variation in the atomic ratio of oxygen to tantalum follows that in the measured refractive index. That is, the larger is the atomic ratio of oxygen to tantalum, the higher is the refractive index of the film.

\section{CONCLUSIONS}

In this study, non-stoichiometric amorphous tantalum oxide films with atomic ratios of oxygen to tantalum less than 2.5 have been grown with the reactive r.f. sputtering technique. It is found that, when the oxygen content is increased with a constant argon partial pressure, both the refractive index and the deposition rate 
increase at smaller oxygen contents and then decrease at larger oxygen contents. On increasing the total sputtering gas pressure, both the refractive index and the deposition rate of the deposited films increase, at lower total pressures, to a peak value and then decrease, at higher total pressures. When the r.f. sputtering power is increased, the refractive index of the film will decrease. Variation of the substrate temperature will not affect the deposition rate of the films significantly, but the refractive index will increase with increased temperature. The RBS analyses show that the deposited films at higher substrate temperatures will be more stoichiometric and closer to the structure of $\mathrm{Ta}_{2} \mathrm{O}_{5}$. However, the XRD measurements show that the structures of the reactively r.f.-sputtered tantalum oxide films are still amorphous rather than crystalline. Different compositions of the deposited tantalum oxide films have been obtained from the RBS analyses. From these analyses, it is found that the deposited tantalum oxide films with larger atomic ratios of oxygen to tantalum will have higher refractive indices.

\section{REFERENCES}

1 R. W. Berry, P. M. Hall and M. T. Harris, Thin Film Technology, Van Nostrand Reinhold, New York, 1968.

2 W. D. Westwood, N. Waterhouse and P. S. Wilox, Tantalum Thin Films, Academic Press, London, 1975.

3 F. Zernike, in T. Tamir (ed.), Integrated Optics, Topics in Applied Physics, Vol. 7, Springer, New York, 2nd end., 1979, Chap. 5.

4 W. M. Paulson, F. S. Hickernell and R. L. Davis, J. Vac. Sci. Technol., 16 (1979) 307.

5 R. L. Davis and F. S. Hickernell, Proc. Soc. Photo-Opt. Instrum. Eng., 408 (1982) 27. 
